\title{
Efficacy of posterior-only decompression, focal clearance, and internal fixation in treatment of thoracic and lumbar tuberculosis.
}

\author{
Xufeng Jia ${ }^{1,2}$, Miao Long ${ }^{1}$, Qingzhong Zhou ${ }^{2}$, Yong Ji', Guangping Huang ${ }^{1}$, Yu Zhou ${ }^{1}$, Daxiong Feng ${ }^{2 *}$ \\ ${ }^{1}$ Department of Orthopaedics, the People's Hospital of Jianyang City, Jianyang Sichuan, PR China \\ ${ }^{2}$ Department of Spine Surgery, the Affiliated Hospital of Southwest Medical University, Luzhou, Sichuan, PR China
}

\begin{abstract}
Introduction: Thoracic and lumbar tuberculosis significantly affect the health of people and there is controversy on the therapeutic method.

Aims: Our objective is to investigate the clinical efficacy of posterior-only decompression, focal clearance, and internal fixation in treatment of thoracic and lumbar tuberculosis.

Methods: Totally 53 eligible patients with thoracic and lumbar tuberculosis were selected and divided into group A (27 patients) and group B (26 patients) according to the admission order. In group A, posterior-only focal clearance, pedicle screw fixation, and fusion of titanium web and the grafted bone were applied; while in group $B$, combined posterior and anterior surgery, namely posterior internal fixation with nail-stick system in the pedicle of vertebral arch to correct abnormalities first and then focal clearance, were applied. The operative time, intra-operative blood losses, drainage time, drainage volumes, and postoperative lengths of stay were compared between the two groups, and the time to fusion of the grafted bone, degree and rate of correction of kyphotic abnormality, and ASIA impairment scale of neurologic function were followed up.

Results: The operative time, intra-operative blood loss, drainage time, drainage volume, and postoperative length of stay in the group $A$ were better than the group B, which were statistically significant (all $\mathbf{p}<\mathbf{0 . 0 5}$ ). All the patients were followed up for 12 months-23 months. At 1 year after surgery, the time to fusion of the grafted bone was not statistically different between two groups $(p>0.05)$, however, the degree and rate of correction of Cobb angle in the group A were better than the group $B$, which were statistically significant (both $p<0.05$ ). After the surgery, the improvement of neurologic function ASIA classification was not statistically different between two groups $(\mathbf{p}>\mathbf{0 . 0 5})$.

Conclusions: Posterior-only decompression, focal clearance, and internal fixation in treatment of thoracic and lumbar tuberculosis have small operation wound and has definite effects of spinal reshaping and fusion.
\end{abstract}

Keywords: Spinal tuberculosis, Posterior decompression, Focal clearance, Internal fixation. Accepted on October 27, 2017

\section{Introduction}

Vertebral body involvement in spinal tuberculosis can cause vertebral body collapse, which causes kyphotic abnormality, nerve injury, and even paraplegia in severe case, significantly affecting the life and work of patients [1-3]. In the spinal tuberculosis patients with existing bone structure destruction or nerve system complications, the effect of antituberculosis drug treatment is limited, and the surgery is required to restore spinal stability [4-6]. Focal clearance, intervertebral bone-graft and internal fixation are the most common surgical regimen, which can be done through anterior, posterior and the combined approaches in one stage or multiple stage surgery [7-10]. At present, there is controversy about the posterior-only decompression and internal fixation [5,11]. 27 thoracic and lumbar tuberculosis patients who received posterior-only decompression, focal clearance, and internal fixation were compared with 26 patients who received combined posterior and anterior surgery, focal clearance, and internal fixation to discuss the efficacy of the former regimen.

\section{Materials and Methods}

\section{Selection of patients}

Inclusion criteria: Patients with thoracic and lumbar spinal tuberculosis and aged 18-70 years old; the involved vertebral body is mainly at single level; the continuous involved vertebral bodies in the skipped spinal tuberculosis is within 1 motor unit; there is big sequestrum or cavity in the damaged vertebral body; spine malformation, instability and dislocation are progressively aggravated; there are obvious clinical 
symptoms and neurologic function injury; the patients agree to participate in this study and sign the informed consent.

Exclusion criteria: Patients with dysfunction of important organs such as heart, liver and kidney who cannot tolerate the surgery; patients with multi-segmental large paraspinal abscess or flowing abscess; Patients complicated with lung tuberculosis received standard chemotherapy for $2 \mathrm{~W}$ and the toxic symptoms are not alleviated; Patients with multi-segment vertebral involvement or severe collapse; Patients with thoracic and lumbar vertebral body trauma or surgical history; Some diseases that can affect the evaluation such as congenital vertebral deformities. This study is approved by the Ethics Committee in our hospital.

\section{Baseline data of patients}

53 patients with thoracic and lumbar tuberculosis who met the criteria were selected, including 29 male patients and 24 female patients. The age was $27-69(45.90 \pm 12.71 \mathrm{y}$ old $)$; the disease course was $1-3(1.42 \pm 0.37 \mathrm{y})$. The tuberculosis location: at thoracic vertebra (T9, T10) in 13 cases, at thoracic and lumbar vertebra (T11-L2) in 16 cases and at lumbar vertebra (L3, L4) in 24 cases. The American Spinal Injury Association (ASIA) impairment scale [12,13]: 6 cases of grade B, 30 cases of grade C, 13 cases of grade D, and 4 cases of grade E. 16 cases had no obvious paraspinal abscess and 37 cases had slight paraspinal abscess; the main manifestation by imaging examination: there was damage or collapse of vertebral body, sequestrum formation, epidural or paraspinal abscess, kyphotic abnormality or spinal cord compression. 53 patients were divided into group A (posterior-only focal clearance, pedicle screw fixation, and fusion of titanium web and the grafted bone) and B (combined posterior and anterior surgery, namely, posterior internal fixation with nail-stick system in the pedicle of vertebral arch to correct abnormalities first, and then focal clearance) according to the admission order. The preoperative baseline data of two groups were not statistically different $(\mathrm{p}>0.05)$.

\section{Preoperative preparation}

The patients received standard anti-tuberculosis medicine treatment, the Erythrocyte Sedimentation Rate (ESR) decreased to $30 \mathrm{~mm} / 1 \mathrm{~h}$, the tuberculosis was effectively controlled, the hypoalbuminemia was corrected and the nutrition supply was sufficient.

\section{Surgical regimen}

Group A: The patients received tracheal intubation and anesthesia. The patients were at prone position. The diseased segment was located by $\mathrm{C}$ arm X-ray machine, the diseased vertebral bodies and upper and lower 2-3 vertebral bodies were exposed layer by layer, and the transverse processes were exposed at both sides. The diseased vertebral body and the transverse process of its upper vertebral body were removed for decompression, and then the pedicle screws were screwed in the upper and lower adjacent normal vertebral bodies. The zygapophysial joints at the severely damaged side of vertebral body were removed to clean the focus, the damaged lesion at anterior vertebral body was cleaned along with the vertebral edge. Curet and Tian's spinal osteotome were used to remove the diseased vertebral body and intervertebral disc. In the patients with paraspinal abscess, the abscess was cleaned forward from intertransverse space, the excised transverse process or ribs based on CT localization. After the lesion was cleaned, hydrogen peroxide and $0.9 \%$ sodium chloride injection were used to repeatedly flush the surgical field. The bone graft bed was reconditioned, a proper amount of iliac bone was made into small bone pieces, and mixed with streptomycin and isoniazid for intervertebral bone grafting; or titanium mesh with a certain length and diameter was taken to be filled with autogenous bone. The fixing rod was reinstalled at the target side, after the kyphotic abnormality correction, vertebral body restoration and internal fixation position were confirmed by X-ray, the surgical field was flushed again. After confirming that there was no spinal cord or nerve root compression, the vertebral plate, zygapophysis and transverse process of diseased segment were fused by bone graft, the drainage tube was place and the incision was sutured.

Group B: The patients received tracheal intubation and anesthesia. The patients were at prone position. First the patients received posterior internal fixation with nail-stick system in the pedicle of vertebral arch to correct abnormalities and then the incision was sutured. After that the patients were at the lateral position, and focal clearance was completed through extra pleuroperitoneal approach by repeatedly flushed by hydrogen peroxide and $0.9 \%$ sodium chloride injection. The bone bed was reconditioned for bone graft, and drainage tubes were placed through anterior and posterior approaches, and then the incision was sutured.

\section{Postoperative management}

The drainage tube was kept for 24-72 h. Infection prevention, nutrition support, and immunity improvement was applied. Triple or quadruple anti-tuberculosis medicine treatment was given. The patients were allowed to start ground activity with thoracic and lumbar support after bed rest for $3 \mathrm{w}$. Neurotrophy and rehabilitation therapy were given to promote neurologic function recovery.

\section{Observation indexes}

The operation time, intra-operative blood loss, drainage time, drainage volume, and postoperative length of stay were recorded. The bone graft fusion time, the corrected degree and rate of kyphotic abnormality and neurological function ASIA scale were followed up.

\section{Statistical analysis}

The data were analysed by SPSS 13.0. The measurement data were presented as $\overline{\mathrm{X}} \pm \mathrm{s}$ and analysed by t-test, the enumeration data were analysed by $\chi^{2}$ test. $\mathrm{p}<0.05$ was considered as statistically significant. 
Efficacy of posterior-only decompression, focal clearance, and internal fixation in treatment of thoracic and lumbar tuberculosis

\section{Results}

\section{The comparison of surgical related indexes between two groups}

As shown in Table 1, the operative time, intra-operative blood loss, drainage time, drainage volume, and postoperative length of stay in the group A were better than the group B, which were statistically significant (all $\mathrm{p}<0.05$ ).

\section{The bone graft fusion time}

All the patients were followed up for 12 months -23 months. The grafted bones were fused in two groups, the time to fusion of the grafted bone in group A was 7.1-9.6 (7.54 \pm 0.49$)$ months and in group B was 7.2-9.5 (7.49 \pm 0.44$)$ months, which were not statistically different between two groups ( $>0.05$ ).

\section{Comparison of kyphotic abnormality correction angle and rate between two groups}

As shown in Table 2, after 1 year the correction degree and rate of Cobb angle in the group A were better than the group B, which were statistically significant (both $\mathrm{p}<0.05$ ).

\section{Comparison of neurologic function ASIA scale before and after surgery between two groups}

As shown in Table 3, 1 y after the surgery the neurologic function ASIA scale was improved compared with before the surgery in two groups, which were statistically different $(p<0.05)$, and after the surgery the neurologic functions was not statistically different between two groups ( $\mathrm{p}>0.05$ ).

The classical cases are shown in Figures 1-3.
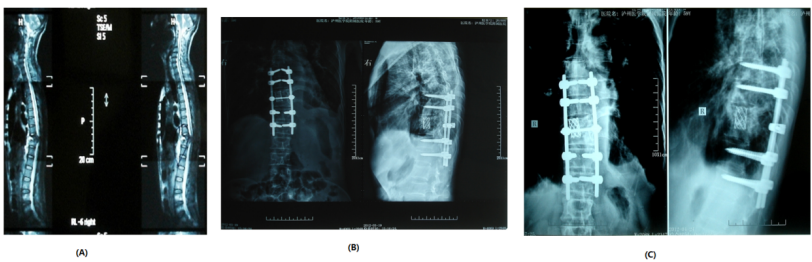

Figure 1. Case 1, male, 59 y old, repeated abdominal pain for $1 y$, which aggravated for $10 \mathrm{~d}$, diagnosed as T10-11 spinal tuberculosis complicated with paravertebral abscess. The patient received posterior-only decompression, focal clearance, and internal fixation and titanium mesh bone grafting. A. Preoperative MRI suggested kyphotic abnormality, $T 7$ vertebral body damage, narrowing of adjacent intervertebral space, paravertebral abscess and sequestrum formation. B. Anteroposterior and lateral X-ray after 3 months, the fixation was at the right position, there was no breakage or shirting, the paravertebral abscess was absorbed and grafted bone was partially fused. C. Anteroposterior and lateral X-ray after 6 months, kyphotic abnormality was corrected, the fixation was at the right position, there was no breakage, loosening or shifting, and grafted bone was fused.
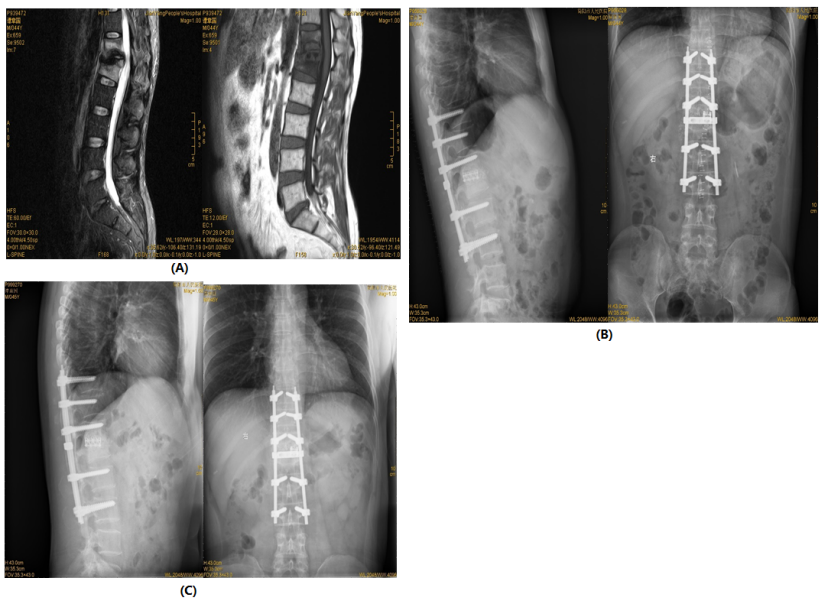

(B)

Figure 2. Case 2, male, 44 y old, lumbago for two years which aggravated 1 month, diagnosed as T12, L1 spinal tuberculosis. The patients received posterior-only decompression, focal clearance, and internal fixation and titanium mesh bone grafting. A. Preoperative MRI suggested that the intervertebral space between T12-L1 was narrowed and damaged, the paravertebral abscess was formed. $B$. Postoperative anteroposterior and lateral X-ray suggested that the fixation was at the right place without loosening or breakage, and there was no titanium mesh shifting. C. Postoperative anteroposterior and lateral X-ray after 1 y suggested that the fixation was at the right place without loosening or breakage, the titanium mesh was at the right place, and the bone graft fused.
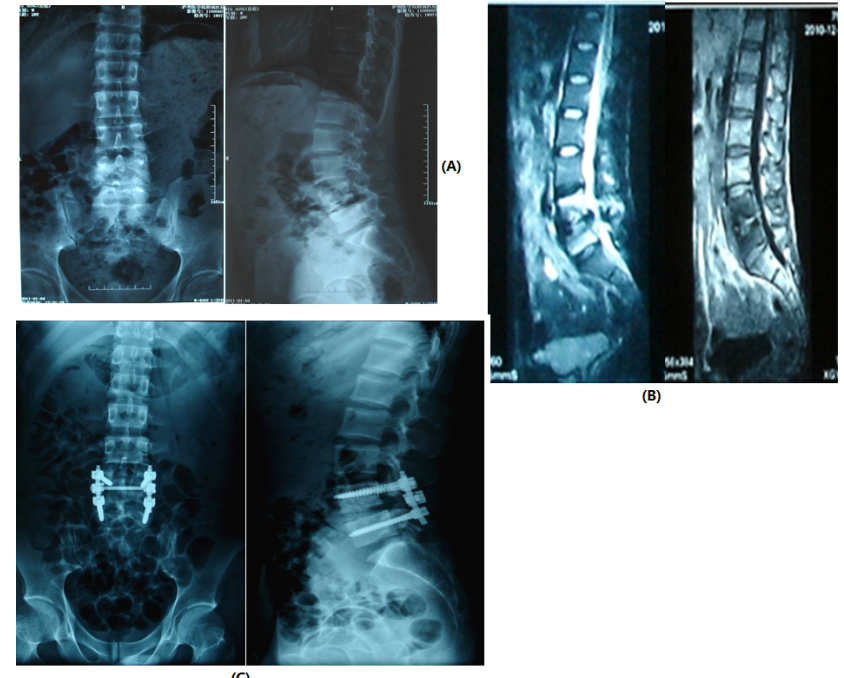

Figure 3. Case 3, male, 39 y old, lumbago for 6 months which gradually aggravated for 1 month, diagnosed as L4, 5 spinal tuberculosis. A. Preoperative anteroposterior and lateral X-ray showed L4, 5 intervertebral space damage and narrowing, the psoas major muscle was swollen. B. Preoperative MRI showed L4, 5 intervertebral space damage, prevertebral abscess, necrotic tissue and intervertebral sequestrum. C. Peoperative anteroposterior and lateral X-ray after 6 months showed the pedicle screw was at the right place, the intervertebral space was clear and there was intervertebral fusion. 


\section{Discussion}

\section{The therapeutic regimen of spinal tuberculosis}

In the recent years, the resurgence of tuberculosis is obvious and the morbidity is increasing year by year due to the increase of floating population, increase of immunosuppressant administration and immunodeficiency patients and the emerging of drug-resisted tubercle bacillus [14-16]. The therapeutic principle of spinal tuberculosis includes thoroughly focal clearance, spinal decompression and recovery, and the reestablishment of spinal column sequence and stability $[13,17,18]$. Anterior-only surgery can more directly clear the lesion, however there are risks such as vessel and ureter injury, and thoracoabdominal cavity infection. Furthermore, in the clinical practice it is found that it tends to cause fixation loosening, collapse, malformation correction failure and nerve compression $[19,20]$. In anterior and posterior surgery, the therapeutic effect is definite due to the anterior focal clearance and bone graft and posterior internal fixation. However, the surgical injury is big and the occurrence rate of kyphotic abnormality is high, which have limited the application. In posterior-only surgery, pedicle screw was used for 3 column fixation, the fixation strength is strong and the spine is fused at $360^{\circ}$, which had obvious advantage in restoring the spinal stability and correcting the kyphotic abnormality [21-23]. Some researchers have reported that in the patients received anterior or posterior internal fixation to treat thoracic and lumbar tuberculosis respectively, the results showed that posterior surgery has more advantages in correcting kyphotic abnormality and maintaining spinal stability [5,24,25]. Some other researchers have shown that a large number of patients with spinal tuberculosis treated by conservative therapy can be self-cured. That is because the control of tuberculosis depends on the anti-tuberculosis drug and improvement of the immune function, and in posterior surgery the lesion can be cleared extensively and the tubercle bacillus focus can be damaged to transfer the tubercle bacillus from resting stage to proliferative stage, which is beneficial to postoperative anti-tuberculosis treatment $[10,26]$. At present, there is controversy about the surgical regimen of thoracic and lumbar tuberculosis $[10,27,28]$.

\section{Efficacy comparison of therapeutic regimens between two groups}

In this study, the patients with thoracic and lumbar tuberculosis received posterior-only surgery and anterior and posteriors surgery respectively, the results showed that the operative time, intra-operative blood loss, drainage time, drainage volume, and postoperative length of stay in the former one were better than the latter one, suggesting that posterior-only surgery causes smaller injury. During $1 \mathrm{y}$ follow up, the time to fusion of the grafted bone was similar, the degree and rate of correction of Cobb angle and ASIA scale were not different between two groups, suggesting that although posterior-only surgery can only relatively thoroughly clear the lesion, it doesn't affect the clinical therapeutic effect. Further results showed that after 1 year, the degree and rate of correction of Cobb angle in group A were better than group B, suggesting that posterior-only surgery is better in correcting malformation and maintaining the stability of spinal mechanic stability. The reason may be that the modified bone fragment, the bone graft implanted between vertebral plates, facet joints and spinous processes through posterior approach reestablished the three-column structure of spine. The subhealthy bone tissue kept during the surgery can transform to normal bone tissue after standard antituberculosis treatment, which provides a basis for spinal stability [29-32].

\section{The advantages and implementation notes}

In this study, the results showed that posterior-only surgery in thoracic and lumbar tuberculosis have the following advantages: focal clearance, intervertebral bone grafting, kyphotic abnormality correction and pedicle screw fixation were all competed in one-stage surgery, it was not necessary to change patient position, the surgical injury was small, the postperative recovery was fast, the length of stay in hospital was shortened and the would infection rate was decreased; the bone graft can be implanted between vertebral bodies, vertebral plates or spinous processes, which can fuse the spinal in $270^{\circ}$ range; The bone grafting was completed under a direct vision, the spinal compression was thorough, the protective effect to spine cord was better and it can also effectively avoid the vessel injury caused bleeding; Pedicle screw system is throughout the whole 3 column spine and the reestablishment stability was good which was beneficial to correct kyphotic abnormality; less contact between internal fixation and tuberculous focus can decrease internal fixation infection and increase the operative successful rate; exposed posterior structure is beneficial for postoperative drainage and promoting recovery; during posterior-only surgery the following points should be paid attention to: the posterior column structure was damaged during the surgery which possibly increased the instability; if the lesion is extensive and the surgical field is limited, surgeons can only operate with surgical equipment and hand feel, thus the lesion cannot be thoroughly cleaned; Posterior decompression can expose the spinal cord, thus the spine cord should be protected during the surgery; the abscess at the surface of psoas major muscle is difficult to be cleared, thus needle puncture aspiration or tube drainage are needed.

However, there are several limitations. Firstly the sample size is relatively small. A larger sample size may be enrolled in the future and the conclusion will be more convincing. Secondly, this study is a single-center study, in the future we will do a multi-center study to verify this conclusion.

In conclusion, posterior-only decompression, focal clearance, bone grafting, internal fixation and kyphotic abnormality correction in the treatment of thoracic and lumbar tuberculosis have small surgical injury and definite effects of spinal reshaping and fusion, and the postoperative recover is fast. 
Efficacy of posterior-only decompression, focal clearance, and internal fixation in treatment of thoracic and lumbar tuberculosis

However, a large-scale follow up and longer therapeutic effect are needed.

Table 1. The comparison of surgical related indexes between two groups.

\begin{tabular}{|c|c|c|c|c|c|}
\hline Group (n) & Operative time (min) & $\begin{array}{l}\text { Intra-operative blood loss } \\
(\mathrm{ml})\end{array}$ & Drainage time $(d)$ & Drainage volume (ml) & $\begin{array}{l}\text { Postoperative length of stay } \\
\text { (d) }\end{array}$ \\
\hline$A(27)$ & $268.90 \pm 25.02$ & $626.27 \pm 54.61$ & $1.45 \pm 0.27$ & $175.44 \pm 24.80$ & $16.55 \pm 3.46$ \\
\hline B (26) & $384.86 \pm 28.47$ & $884.60 \pm 74.82$ & $2.93 \pm 0.35$ & $393.12 \pm 32.74$ & $21.30 \pm 4.54$ \\
\hline $\mathrm{t}$ value & 6.774 & 8.905 & 4.817 & 6.462 & 8.162 \\
\hline$P$ value & $<0.05$ & $<0.05$ & $<0.05$ & $<0.05$ & $<0.05$ \\
\hline
\end{tabular}

Table 2. The comparison of Cobb angle before and after surgery between two groups $(\bar{x} \pm s)$.

\begin{tabular}{clll}
\hline Group (n) & Cobb $\left({ }^{\circ}\right)$ & $\begin{array}{l}\text { Corrected } \\
\text { angle }\left({ }^{\circ}\right)\end{array}$ & $\begin{array}{l}\text { Correction } \\
\text { rate (\%) }\end{array}$ \\
\cline { 2 - 3 } & $\begin{array}{l}\text { Before } \\
\text { surgery }\end{array}$ & $\begin{array}{l}1 \text { y after } \\
\text { surgery }\end{array}$ & \\
& &
\end{tabular}

\begin{tabular}{lllll}
\hline $\mathrm{A}(27)$ & $42.34 \pm 7.11$ & $11.60 \pm 6.68$ & $31.57 \pm 6.92$ & $76.22 \pm 5.86$ \\
\hline $\mathrm{B}(26)$ & $41.83 \pm 7.17$ & $26.25 \pm 6.80$ & $15.39 \pm 7.85$ & $42.59 \pm 7.31$ \\
\hline $\mathrm{t}$ value & 1.528 & 7.904 & 8.41 & 10.332 \\
\hline $\mathrm{P}$ value & $>0.05$ & $<0.05$ & $<0.05$ & $<0.05$ \\
\hline
\end{tabular}

Table 3. Comparison of neurologic function ASIA scale before and after surgery between two groups (case).

\begin{tabular}{|c|c|c|c|c|c|c|c|c|c|c|}
\hline \multirow[t]{2}{*}{ Time point } & \multicolumn{5}{|c|}{ Group A (n=27) } & \multicolumn{5}{|c|}{ Group $B(n=26)$} \\
\hline & Grade A & Grade B & Grade C & Grade D & Grade E & Grade A & Grade B & Grade C & Grade D & Grade E \\
\hline $\begin{array}{l}\text { Before } \\
\text { surgery }\end{array}$ & 0 & 2 & 16 & 7 & 2 & 0 & 4 & 14 & 6 & 2 \\
\hline After surgery & 0 & 0 & 0 & 2 & 25 & 0 & 0 & 0 & 3 & 23 \\
\hline$x^{2}$ & 4.858 & & 4.386 & & & & & & & \\
\hline$P$ & $<0.05$ & & $<0.05$ & & & & & & & \\
\hline
\end{tabular}

\section{References}

1. Kasundra GM, Sood I, Bhushan, B, Bhargava AN, Shubhkaran K. Distal cord-predominant longitudinally extensive myelitis with diffuse spinal meningitis and dural abscesses due to occult tuberculosis: a rare occurrence. J Pediatr Neurosci 2016; 11: 77-79.

2. Sudprasert W, Choovongkomol K, Piyapromdee U, Leownorasate $M$. Impact on neurological recovery of transforaminal debridement and interbody fusion versus transpedicular decompression in combination with pedicle screw instrumentation for treating thoracic and lumbar spinal tuberculosis. Asian Spine J 2016; 10: 543-552.

3. Wang YX, Zhang HQ, Tang MX, Guo CF, Deng A, Wu JH, Liu JY, Deng Z, Chen J. One-stage posterior focus debridement, interbody grafts, and posterior instrumentation and fusion in the surgical treatment of thoracolumbar spinal tuberculosis with kyphosis in children: a preliminary report. Childs Nerv Syst 2016; 32: 1495-1502.

4. Huang J, Zhang H, Zeng K, Gao Q. The clinical outcomes of surgical treatment of noncontiguous spinal tuberculosis: a retrospective study in 23 cases. PLoS One 2014; 9: 93648.
5. Yang P, He X, Li H, Zang Q, Yang B. Clinical efficacy of posterior versus anterior instrumentation for the treatment of spinal tuberculosis in adults: a meta-analysis. J Orthop Surg Res 2014; 9: 10.

6. Zhang P, Shen Y, Ding WY, Zhang W, Shang Z. The role of surgical timing in the treatment of thoracic and lumbar spinal tuberculosis. Arch Orthop Trauma Surg 2014; 134: 167-172.

7. Hu J, Li D, Kang Y, Pang X, Wu T, Duan C, Cao Y. Active thoracic and lumbar spinal tuberculosis in children with kyphotic deformity treated by one-stage posterior instrumentation combined anterior debridement: preliminary study. Eur J Orthop Surg Traumatol 2014; 24: 221-229.

8. Vamvanij V, Ruangchainikom M, Thanapipatsiri S, Pichaisak W. The outcomes of combined posterior instrumentation and anterior radical debridement with fusion for multilevel spinal tuberculosis. J Med Assoc Thai 2014; 97: 50-55.

9. Zhang HQ, Hu X, Yin X, Chen Y. One-stage combined anterior-posterior approach treatment of multiple cervicothoracic spinal tuberculosis with kyphosis. Int Orthop 2015; 39: 1605-1610. 
10. Zhong W, Xiong G, Wang B, Lu C, Dai Z, Lv G. Surgical management for thoracic spinal tuberculosis posterior only versus anterior video-assisted thoracoscopic surgery. PLoS One 2015; 10: 0119759.

11. Liu J, Wan L, Long X, Huang S, Dai M, Liu Z. Efficacy and safety of posterior versus combined posterior and anterior approach for the treatment of spinal tuberculosis: a meta-analysis. World Neurosurg 2015; 83: 1157-1165.

12. Luo C, Wang X, Wu P, Ge L, Zhang H, Hu J. Single-stage transpedicular decompression, debridement, posterior instrumentation, and fusion for thoracic tuberculosis with kyphosis and spinal cord compression in aged individuals. Spine J 2016; 16: 154-162.

13. Soares Do Brito J, Tirado A, Fernandes P. Surgical treatment of spinal tuberculosis complicated with extensive abscess. Iowa Orthop J 2014; 34: 129-136.

14. Cavalli Z, Ader F, Valour F, Saison J, Boussel L, Dumitrescu O, Perpoint T, Chidiac C, MayT, Ferry T, Lyon TBS. Clinical presentation, diagnosis, and bacterial epidemiology of peritoneal tuberculosis in two university hospitals in France. Infect Dis Ther 2016; 5: 193-199.

15. Elmadhoun WM, Noor SK, Bushara SO, Ahmed EO, Mustafa H, Sulaiman AA, Almobarak AO, Ahmed MH. Epidemiology of tuberculosis and evaluation of treatment outcomes in the national tuberculosis control programme, River Nile state, Sudan, 2011-2013. East Mediterr Health J 2016; 22: 95-102.

16. Krsulovic FA, Lima M. Tuberculosis epidemiology at the country scale: self-limiting process and the HIV effects. PLoS One 2016; 11: 0153710.

17. Li J, Li XL, Zhou XG, Zhou J, Dong J. Surgical treatment for spinal tuberculosis with bilateral paraspinal abscess or bilateral psoas abscess: one-stage surgery. J Spinal Disord Tech 2014; 27: 309-314.

18. Rajasekaran S, Kanna RM, Shetty AP. Pathophysiology and Treatment of Spinal Tuberculosis. JBJS Rev 2014; 2.

19. Wang B, Lv G, Liu W, Cheng I. Anterior radical debridement and reconstruction using titanium mesh cage for the surgical treatment of thoracic and thoracolumbar spinal tuberculosis: minimium five-year follow-up. Turk Neurosurg 2011; 21: 575-581.

20. Wang X, Pang X, Wu P, Luo C, Shen X. One-stage anterior debridement, bone grafting and posterior instrumentation vs. single posterior debridement, bone grafting, and instrumentation for the treatment of thoracic and lumbar spinal tuberculosis. Eur Spine J 2014; 23: 830-837.

21. Sahoo MM, Mahapatra SK, Sethi GC, Dash SK. Posterioronly approach surgery for fixation and decompression of thoracolumbar spinal tuberculosis: a retrospective study. J Spinal Disord Tech 2012; 25: 217-223.

22. Talu U, Gogus A, Ozturk C, Hamzaoglu A, Domanic U. The role of posterior instrumentation and fusion after anterior radical debridement and fusion in the surgical treatment of spinal tuberculosis: experience of 127 cases. J Spinal Disord Tech 2006; 19: 554-559.
23. Zhang HQ, Lin MZ, Shen KY, Ge L, Li JS, Tang MX, Wu JH, Liu JY. Surgical management for multilevel noncontiguous thoracic spinal tuberculosis by single-stage posterior transforaminal thoracic debridement, limited decompression, interbody fusion, and posterior instrumentation (modified TTIF). Arch Orthop Trauma Surg 2012; 132: 751-757.

24. Pinglin Y, Xijing H, Haopeng L, Quanjin Z, Baohui Y. Clinical efficacy of posterior versus anterior instrumentation for the treatment of spinal tuberculosis in adults: a meta-analysis. J Orthop Surg Res 2015; 10: 40.

25. Zhang HQ, Li JS, Zhao SS, Shao YX, Liu SH, Gao Q, Lin MZ, Liu JY, Wu JH, Chen J. Surgical management for thoracic spinal tuberculosis in the elderly: posterior only versus combined posterior and anterior approaches. Arch Orthop Trauma Surg 2012; 132: 1717-1723.

26. Shen X, Liu H, Wang G, Pang X, Luo C, Zeng H, Xu Z. The role of single-stage posterior debridement, interbody fusion with titanium mesh cages and short-segment instrumentation in thoracic and lumbar spinal tuberculosis. J Neurosurg Sci 2015.

27. He QY, Xu JZ, Zhou Q, Luo F, Hou T, Zhang Z. Treatment effect, postoperative complications, and their reasons in juvenile thoracic and lumbar spinal tuberculosis surgery. J Orthop Surg Res 2015; 10: 156.

28. Yao Y, Zhang H, Liu M, Liu H, Chu T, Tang Y, Zhou Y. Prognostic factors for recovery of patients after surgery for thoracic spinal tuberculosis. World Neurosurg 2017; 105: 327-331.

29. Kumar MN, Joseph B, Manur R. Isolated posterior instrumentation for selected cases of thoraco-lumbar spinal tuberculosis without anterior instrumentation and without anterior or posterior bone grafting. Eur Spine J 2013; 22: 624-632.

30. Liu JM, Chen XY, Zhou Y, Long XH, Chen WZ, Liu ZL, Huang SH, Yao HQ. Is nonstructural bone graft useful in surgical treatment of lumbar spinal tuberculosis? A retrospective case-control study. Medicine (Baltimore) 2016; 95: 4677.

31. Mathur S, Aswani Y, Sankhe SS, Hira PR. Aneurysmal bone cyst of thoracic spine mimicking spinal tuberculosis. J Craniovertebr Junction Spine 2011; 2: 99-101.

32. Shen X, Huang X, Xiao S, Liu H, Zhang Y, Xiang T, Wang G, Sheng B, Huang S, Liu X. Surgical treatment of selected patients with multilevel contiguous thoracolumbar spinal tuberculosis by only posterior instrumentation without any bone fusion. Int J Clin Exp Med 2015; 8: 18611-18619.

\section{*Correspondence to}

Daxiong Feng

Department of Spine Surgery

The Affiliated Hospital of Southwest Medical University

PR China 